\title{
Transcriptomic signatures of schizophrenia revealed by dopamine perturbation in an ex vivo model
}

\author{
Jubao Duan ${ }^{1,2}$, Harald H. H. Göring ${ }^{3}$, Alan R. Sanders ${ }^{1,2}$, Winton Moy ${ }^{1,2}$, Jessica Freda', Eugene I. Drigalenko ${ }^{4}$, Mark Kos $^{3}$, \\ Deli $\mathrm{He}^{1}$ and Pablo V. Gejman ${ }^{1,2}$ MGS
}

\begin{abstract}
The dopaminergic hypothesis of schizophrenia (SZ) postulates that dopaminergic over activity causes psychosis, a central feature of SZ, based on the observation that blocking dopamine (DA) improves psychotic symptoms. DA is known to have both receptor- and non-receptor-mediated effects, including oxidative mechanisms that lead to apoptosis. The role of DA-mediated oxidative processes in SZ has been little studied. Here, we have used a cell perturbation approach and measured transcriptomic profiles by RNAseq to study the effect of DA exposure on transcription in B-cell transformed lymphoblastoid cell lines (LCLs) from 514 SZ cases and 690 controls. We found that DA had widespread effects on both cell growth and gene expression in LCLs. Overall, 1455 genes showed statistically significant differential DA response in SZ cases and controls. This set of differentially expressed genes is enriched for brain expression and for functions related to immune processes and apoptosis, suggesting that DA may play a role in SZ pathogenesis through modulating those systems. Moreover, we observed a non-significant enrichment of genes near genome-wide significant SZ loci and with genes spanned by SZ-associated copy number variants (CNVs), which suggests convergent pathogenic mechanisms detected by both genetic association and gene expression. The study suggests a novel role of DA in the biological processes of immune and apoptosis that may be relevant to SZ pathogenesis. Furthermore, our results show the utility of pathophysiologically relevant perturbation experiments to investigate the biology of complex mental disorders.
\end{abstract}

\section{Introduction}

Schizophrenia (SZ) is a severe brain disorder with $0.5-1 \%$ prevalence and $\sim 80 \%$ heritability estimated from twin studies ${ }^{1}$. The primary evidence for the hyperdopaminergic hypothesis of SZ rests on the observation that psychotogenic stimulants such as methamphetamines lead to elevated brain dopamine (DA) levels and can cause

Correspondence: MGSJubao Duan (jduan@northshore.org) or

Pablo V. Gejman (pgejman@northshore.org)

${ }^{1}$ Center for Psychiatric Genetics, NorthShore University HealthSystem,

Evanston, IL, USA

${ }^{2}$ Department of Psychiatry and Behavioral Neuroscience, University of Chicago,

Chicago, IL, USA

Full list of author information is available at the end of the article.

These authors contributed equally: Jubao Duan, Harald H. H. Göring

Members of the Molecular Genetics of Schizophrenia (MGS) Collaboration are listed at the end of the paper.

\section{(c) The Author(s) 2018}

(c) (i) Open Access This article is licensed under a Creative Commons Attribution 4.0 International License, which permits use, sharing, adaptation, distribution and reproduction c. in any medium or format, as long as you give appropriate credit to the original author(s) and the source, provide a link to the Creative Commons license, and indicate if changes were made. The images or other third party material in this article are included in the article's Creative Commons license, unless indicated otherwise in a credit line to the material. If material is not included in the article's Creative Commons license and your intended use is not permitted by statutory regulation or exceeds the permitted use, you will need to obtain permission directly from the copyright holder. To view a copy of this license, visit http://creativecommons.org/licenses/by/4.0/. shown that amphetamine-induced increases in DA response are correlated with positive symptoms of SZ (e.g., hallucinations and delusions) ${ }^{11-13}$. Recent SZ genome-wide association studies (GWAS) suggest an association between common variants at the dopamine receptor D2 (DRD2) locus and $\mathrm{SZ}^{14}$. However, DA is also known to have non-receptor mediated functions, e.g., through DA autoxidation upon exposure to air or oxygen, a process that contributes to DA neuron loss in Parkinson's disease (PD) and other neurodegenerative disorders involving DA neurotransmission ${ }^{15,16}$. 
While classical hypotheses of SZ etiology focus on neuronal origins of the disease, the strongest and most replicable SZ-GWAS finding is at the extended major histocompatibility complex (xMHC) region ${ }^{14,17-21}$ that is known to contain many genes playing important roles in the immune system. Most recently, common alleles of the $\mathrm{xMHC}$ region immune system gene, complement component $4 A(C 4 A)$, have been shown to be able to explain much of the xMHC region GWAS signal, and $C 4 A$ showed increased expression in SZ postmortem brains ${ }^{22}$. Even without considering the xMHC region, GWASimplicated genes are also enriched in key immune processes such as TGF- $\beta$ signaling, B-cell activation, and Tcell activation ${ }^{14}$. Cytokines play roles in cytotoxicity and apoptosis, as well as influencing DA and other neurotransmitter systems ${ }^{23,24}$ that are implicated in the pathophysiology of SZ. Anti-inflammatory agents, such as celecoxib and aspirin, are reported to ameliorate some psychotic symptoms $^{23}$, and classic antipsychotics affect microglial cells and astrocytes in the central nervous system (CNS) partly through the modulation of the expression of cyclo-oxygenase-2 $(\mathrm{COX}-2)^{25,26}$. There is also growing evidence from clinical studies with COX-2 inhibitors that points to favorable effects of antiinflammatory therapy in $\mathrm{SZ}^{25,26}$. Most recently, type I interferon (IFN) was shown to activate the synapse pruning function of microglia in lupus-prone mice, suggesting a mechanism underlying the prevalent neuropsychiatric conditions in patients with systemic lupus erythematosus ${ }^{27}$.

We hypothesized that some pathogenic effects of DA may be mediated through non-receptor mechanisms, and that some of these effects may be detectable in cells from a primary immune tissue. Lymphoblastoid cell lines (LCL) collections are the most accessible and sizable samples available for functional studies. Given that a large proportion of gene expression signatures are shared between different tissues ${ }^{28-35}$, we have used LCLs as a model to gain insights on SZ biology ${ }^{36-38}$. Here, we have analyzed RNAseq data of 1204 LCLs derived from 514 Europeanancestry (EA) SZ cases and 690 controls in the absence or presence of DA, and have identified different effects of DA on transcriptomic profiles between SZ cases and controls.

\section{Materials and methods Samples}

The initially processed RNAseq sample consisted of 515 SZ cases and 692 controls, and the final analyzed sample contained $514 \mathrm{SZ}$ cases and 690 controls after excluding three expression outliers (see QC below). These EA subjects are from the GWAS- and CNV-studied portion of the MGS collection ${ }^{17,20,39}$ and have previously been reported on for their transcriptomics only at the baseline (unstimulated) condition ${ }^{37}$. There are 639 males (263 cases and 376 controls) and 565 females (251 cases and 314 controls). Detailed phenotypic data have been previously described ${ }^{40}$. Accession numbers from the database of Genotypes and Phenotypes (dbGaP) include phs000775, phs000021, and phs000167. The NorthShore University HealthSystem Institutional Review Board approved the study.

\section{Cell culture and RNA preparation}

LCLs of the study sample were derived at Rutgers University Cell and DNA Repository (RUCDR) ${ }^{36}$. For each LCL, we measured EBV (viral) load (copy number), viable cell count (to index growth rate), and ATP level (to index energy status) at cell harvest (for use as covariates in expression analyses), which are known to have an effect on gene expression in LCLs ${ }^{41}$. For the initially processed $515 \mathrm{SZ}$ cases and 692 controls, RNAseq was carried out in five large batches; further detailed methodology was previously described ${ }^{37}$. For DA perturbation (the pilot on four LCLs and the large-scale RNAseq samples), we grew cells in independent wells (on 6-well plates) in the presence or absence of DA at indicated concentrations. DA perturbation lasted $24 \mathrm{~h}$. To block DA effects, we pretreated the cells with the DA receptor antagonists for $6 \mathrm{~h}$ before adding DA to the cell culture medium. These DA blockers included: D1-like receptor (D1 or D5) antagonist SCH23390 (200 nM; 100-fold saturation concentration $^{42}$ ) and D2-like receptor (D2, D3, or D4) antagonist spiperone $(200 \mathrm{nM} ; \sim 100$-fold saturation concentration $^{43}$ ). We purchased DA, SCH23390, and spiperone from Sigma-Aldrich. We included batch as a possible confounding variable in the analysis, i.e., as a covariate.

\section{RNAseq and data processing}

RNA sequencing was carried out at the University of Minnesota Genomics Center (UMGC) on an Illumina HiSeq2000 at a depth of $\sim 10 \mathrm{M}$ reads/sample. RNAseq data for baseline and DA-stimulated samples were processed as previously described ${ }^{37,38}$. We aligned the 50 -bp single reads to the human reference gene map (Gencode v20) using the mapping tool Tophat v2.0.5 ${ }^{44}$, allowing for two mismatches. We counted the raw reads by using the HTseq-count script (www-huber.embl.de/users/anders/ HTSeq/doc/overview.html $)^{45}$ and calculated gene level expression as RPKM ${ }^{46}$ based on the exon model of the longest transcript of a gene (Gencode v20). We then quantile-normalized gene level RPKMs to help account for batch/run bias ${ }^{38}$.

\section{Quality control}

For RNAseq data quality control (QC), we examined the mean Pearson correlations of gene level RPKM among 10 
technical replicates (i.e., same RNA) and among 16 biological replicates (independent cell cultures of the same $\mathrm{LCL})^{38}$. To capture genes with possible changes from or to very-low expression values upon DA stimulation, we analyzed all the genes $(N=21,043)$ with $R P K M>0$ in at least $50 \%$ of either baseline or DA-stimulated samples. All samples included in the RNAseq had $>6 \mathrm{M}$ mappable reads. We performed initial sample QC by: (1) checking for consistency between expression levels of chromosome $\mathrm{X}(X I S T)$ and chromosome $\mathrm{Y}$ genes (RPS4Y1, ZFY, USP9Y, DDX3Y, UTY, KDMSD, and EIF1AY) vs. reported sex, and (2) by comparing RNAseq-called genotypes (using SAMtools mpileup function ${ }^{47}$, requiring $>8$ reads at a called SNP site) with previous GWAS SNP genotypes (Affymetrix 6.0$)^{17,20}$ for a panel of 175 informative $\mathrm{SNPs}^{17,20}$. The initial sample QC left us with $515 \mathrm{SZ}$ cases and 692 controls. We carried out additional QC analyses to assure no systematic expression bias to either cases or controls, no batch effects, and to identify any potential outlier samples. We first compared the percentage of cases and controls that express each gene (i.e., sample completion rate) at baseline or DA stimulation condition (Fig. S1A-B). Under both baseline and DA-stimulation conditions, the sample completion rate (i.e., proportion of samples with RPKM $>0$ ) in SZ cases and controls were highly correlated $(R=0.99$; Fig. S1). Genes with higher sample completion rate differences between DA and baseline conditions tended to have lower expression values. However, such sample completion rate differences in cases and in controls were highly correlated $(R=0.91$ for all the tested genes, and $R=0.98$ for the subset of genes tested for SZ-associated differential DA response; Fig. S1). Furthermore, the DA-induced gene expression FCs in cases and controls are also well matched (i.e., did not show significant bias to either cases or controls; Fig. S2). Comparing expression correlations among all samples further identified two samples appearing to be "outliers" under the DA stimulation condition (Fig. S3A-B). The same two outliers were also among the three outliers identified by expression PCA (Fig. S3C-D). Both cases and controls appeared to be evenly distributed in a single big cluster in all the plots of expression PCs, except for those outlier samples (Fig. S3C-D). After excluding those three outliers (1 case and 2 controls), a total of 514 SZ cases and 690 controls remained for DA-perturbed differential gene expression analyses.

\section{Differential gene expression analyses}

We first tested differential gene expression before and after DA stimulation. To capture genes that showed very different expression levels in the absence or presence of DA, we considered those with RPKM $>0$ in $\geq$ $50 \%$ of baseline and/or DA-stimulated samples as expressed genes $(N=21,043)$. The rationale of using a more relaxed expression cut-off $(50 \%)$ here vs. a previously used cut-off $(80 \%)^{38}$ was to capture genes that might be expressed at a very-low level under either baseline or DA-stimulated condition, but potentially elevated in the other condition. For both baseline and DA-stimulated expression data, we regressed out the possible effects of affection status, sex, age, cell counts, EBV load, ATP level, genotypic ancestry principal components (PCs 1-5), and sequencing batch (5 batches). Although the cell counts and ATP levels at baseline conditions were just moderately correlated with the same measurements under DA stimulation condition $(R=0.60)$, we included the cell counts and ATP levels at both conditions in the regression analyses, which actually gave very similar results to the analysis when only baseline cell counts and ATP levels were regressed out (Fig. S4). The residuals were used in a paired Student's $t$-test to identify genes showing differential expression between baseline and DAstimulated conditions. FC for a gene was calculated as the ratio of its gene expression value in the presence of DA vs. baseline. The significance of the differential expression was FDR adjusted.

To identify genes that differ in expression level between SZ cases and controls (at either baseline or after DA stimulation), we first log2 (RPKM value +1 )-transformed the expression values, and then used standard multiple linear regression analysis to regress out all the above-listed covariates (except for affection status). The resulting residuals were rank-normalized (to ensure a normal distribution), and are the covariate-adjusted normalized expression values at baseline and upon DA stimulation. We then performed a single linear regression analysis of SZ status (independent variable) on these residuals (dependent variable) to identify the genes whose expression differs between cases and controls at either baseline or upon DA perturbation. The linear regression model is ( $\mathrm{E}$ (rank-normalized residualized expression of gene $\mathrm{X})=$ beta_0 + beta_ $1 \times$ affection status + epsilon). The same approach, but with one additional step, was used to identify genes showing differential DA response between SZ cases and controls. Namely, we subtracted the covariate-adjusted normalized expression values at baseline from the DA state, to obtain the covariateadjusted expression response of DA perturbation. Subsequently, affection status was regressed against these response variables, using a single linear regression model as above. We also explored the effect of regressing out the top 5 expression PCs, after already regressing out the previously mentioned known covariates, in the linear regression analyses, as a check for analytical robustness, in particular the possibility that the selected measured covariates may not have adequately captured all potentially existing confounder effects, which very well might be tagged by top PCs. 


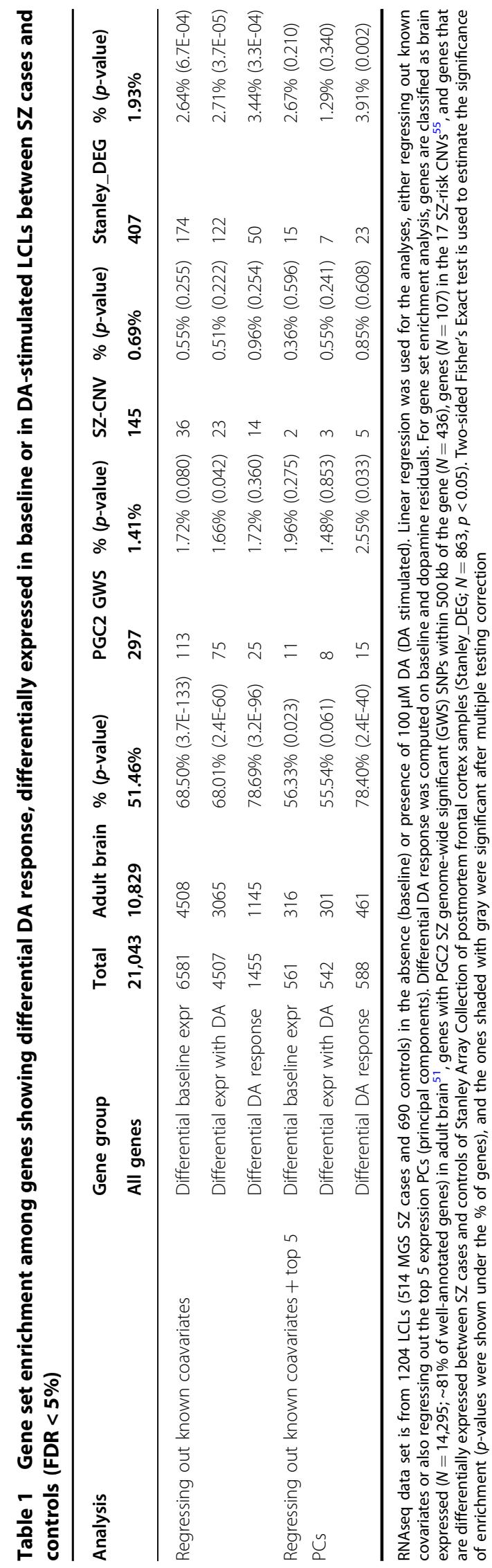

\section{Gene ontology and gene set enrichment analyses}

We used the DAVID tool ${ }^{48}$ and WebGestalt ${ }^{49}$ for GOterm enrichment analyses, with all the genes expressed in LCLs as the background gene set. REVIGO ${ }^{50}$ was used to cluster and visualize the enriched GO-terms. Because of the large number of genes showing differential expression upon DA stimulation ( $>90 \%$; Fig. 3), for GO-term enrichment analysis we selected a subset of genes that showed relatively larger magnitudes presumably representing more likely meaningful biological changes. Based on the distribution of all FCs, we used an arbitrary cut-off of 1SD and included all the genes with FCs of $<0.88$ or $>1.23(N=3756)$, representing $\sim 20 \%$ expression changes upon DA stimulation. For SZ-associated differentially expressed genes, we used $\mathrm{FDR}<5 \%$ to select genes for GO-term enrichment analyses. We used all the LCLexpressed genes in our data set as a reference gene list for enrichment analyses.

For enrichment analysis of specific gene sets, we assembled different gene sets relevant to SZ pathogenesis based on public databases and literature searches as we described $^{38}$, including 14,295 adult brain-expressed genes accounting for $\sim 81 \%$ of well-annotated protein-coding genes $^{51}, 227 \mathrm{SZ}-\mathrm{CNV}$ genes within the $17 \mathrm{SZ}$-associated $\mathrm{CNVs}^{39,52-55}, 435$ genes with SNPs (within $500 \mathrm{~kb}$ ) showing genome-wide significant (GWS) association to SZ in the Psychiatric Genomics Consortium (PGC2) sample $^{14}$, and 863 genes that were differentially expressed $(P<0.05)$ between SZ cases and controls in the Stanley Array Collection of postmortem frontal cortex samples (www.stanleygenomics.org) (Table S8). For genes showing SZ-associated differential DA response, or differential expression at baseline or in the DA-stimulated condition, we counted the number of genes in each gene set. Using all the LCL-expressed genes in each gene set as the denominator, we then estimated the enrichment of each category of differential expressed genes in a preassembled gene set (Table 1). We evaluated the significance of the gene set enrichment using Fisher's exact test (two-sided).

\section{Results}

To investigate the impact of DA exposure on LCLs, we first explored which concentration of DA showed robust effects in LCLs. Subsequently, we examined the transcriptomic profiles associated with DA exposure to identify expression responses associated with SZ (Fig. 1).

\section{DA effects on LCL growth and gene expression}

We tested different DA concentrations in four LCLs of control subjects. We found that DA, at a commonly used concentration range of $100-150 \mu \mathrm{M}^{56-58}$, started to show an inhibitory effect on LCL growth by $\sim 20 \%$ (no effect with smaller DA concentrations) (Fig. 2a), an effect 


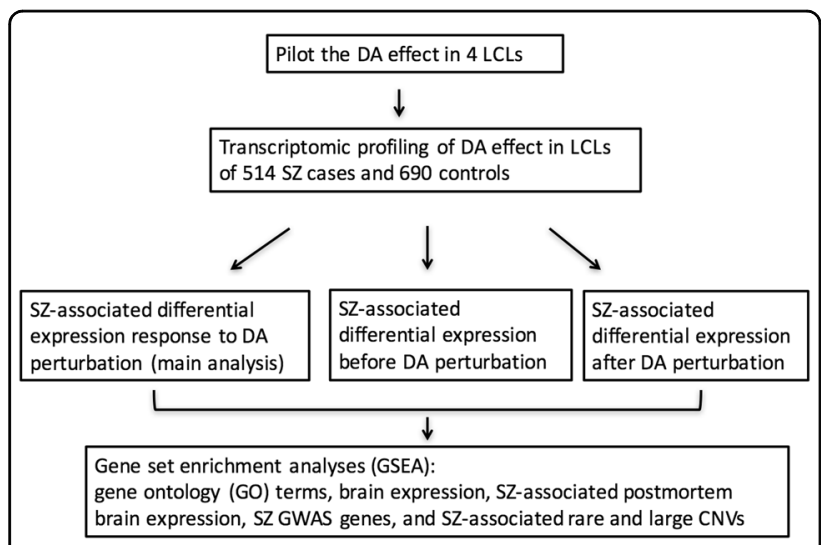

Fig. 1 Flow chart of the study design. SZ schizophrenia, GWAS genome-wide association study, CNV copy number variation, DA dopamine, LCL lymphoblastoid cell line

probably related to an apoptotic effects of $\mathrm{DA}^{59}$. At higher concentrations $(1000 \mu \mathrm{M} \mathrm{DA})$, which is near the estimated DA concentration $(1.6 \mathrm{mM})$ in rat brain synaptic cleft $^{60}, \sim 80 \%$ of the LCLs were dead after $24 \mathrm{~h}$ of exposure (Fig. 2a). Pre-treating LCLs with DA receptor antagonists before applying DA did not block the overall DA effect (Fig. 2b), which was consistent with our observation that LCLs did not show significant expression of DA receptors (Reads Per Kilobase of transcript per Million mapped reads, RPKM $<0.1)$ in LCLs. In the baseline condition, 23,966 genes were expressed in all four LCLs (RPKM $>0$ ). Approximately $13 \%(n=2,999)$ of the genes showed significant expression changes in the presence of $100 \mu \mathrm{M}$ DA (nominal $P<0.05$; paired Student's $t$-test). As expected, a lower DA concentration $(1 \mu \mathrm{M})$ showed a substantially smaller magnitude of gene expression changes (Fig. 2b). As above, the DA effects were not blocked with DA receptor antagonists (Table S1). We decided to use a DA concentration of $100 \mu \mathrm{M}$ in our DA perturbation experiment with a large sample, since this concentration achieved a widespread impact on gene expression with limited cell death.

We next studied the gene expression profiles of 1207 LCLs (515 MGS SZ cases and 692 controls) in the absence (baseline) or presence (DA-stimulated) of $100 \mu \mathrm{M}$ DA. A total of 21,043 genes were expressed $(\mathrm{RPKM}>0)$ in at least $50 \%$ of either baseline or DA-stimulated samples. We found no differences in the proportion of case and control samples expressing a given gene (Fig. S1) or for the expression fold change (FC) upon DA stimulation (Fig. S2). We also identified three samples that were outliers which we removed from further analyses (Fig. S3). The analysis of 1204 clean samples (514 SZ cases and 690 controls) showed that $91 \%(19,085)$ of all the expressed genes were responsive to DA (FDR $<5 \%$; paired Student's $t$-test; Fig. 3a and Table S2). The FCs were small in most

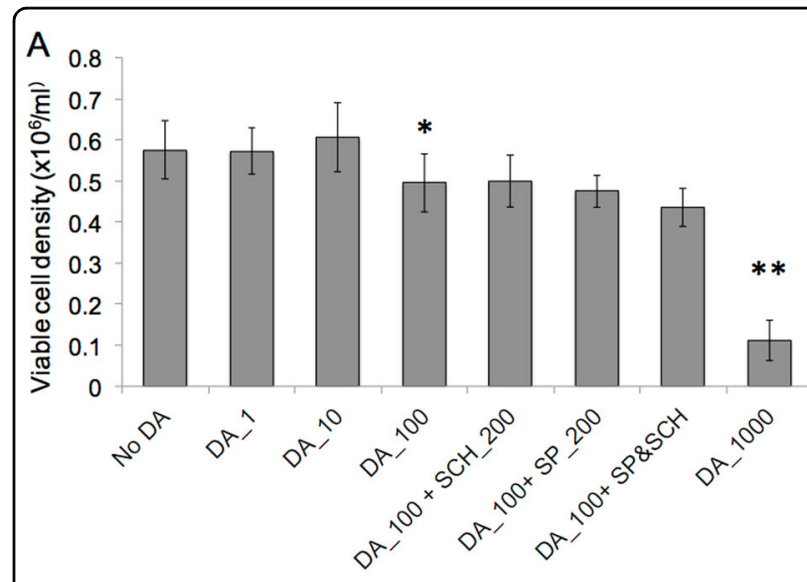

B

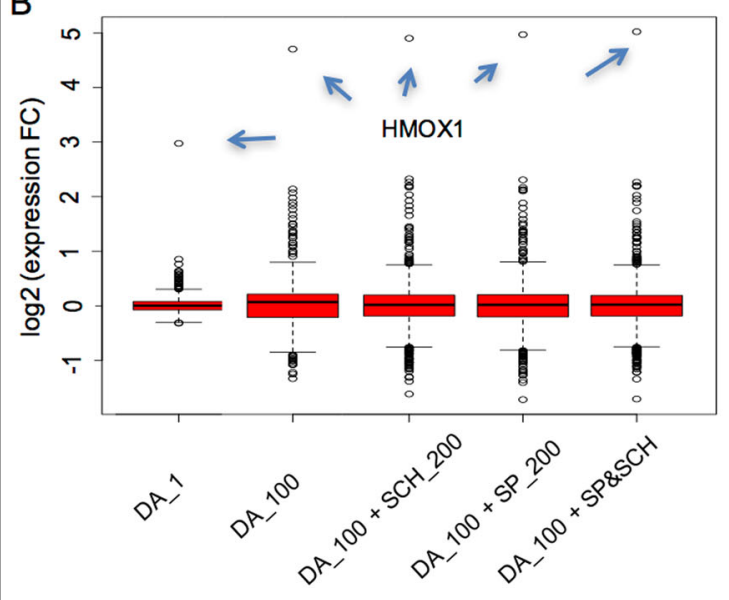

Fig. 2 Dosage effect of dopamine (DA) on cell growth and genome-wide expression of $\mathbf{4}$ LCLs. a DA effect on cell growth after $24 \mathrm{~h}$ of DA treatment $(1 \mu \mathrm{M}, 10 \mu \mathrm{M}, 100 \mu \mathrm{M}$, and 1,000 $\mu \mathrm{M}) .100 \mu \mathrm{M}$ DA started to show a significant inhibitory effect on cell growth, and most cells were dead at $1000 \mu \mathrm{M}$ DA. Pre-treatment of the cells with the D1 receptor antagonist SCH23390 (200 nM) and/or the D2 receptor antagonist Spiperone (SP; $200 \mathrm{nM}$ ) for $6 \mathrm{~h}$ did not block the inhibitory effect of DA on cell growth. ${ }^{*} P<0.05$ and ${ }^{*} P<0.01$ were derived from two-sided paired Student's $t$-test. $\mathbf{b}$ Box plot of DA effect on genome-wide expression. $100 \mu \mathrm{M}$ DA showed a substantially stronger effect than $1 \mu \mathrm{M}$ DA, but pre-treatment of the cells with D1 or D2 receptor antagonists did not reverse the effect of DA. Plotted are 2999 genes that showed significant expression changes after DA

stimulation $(100 \mu \mathrm{M})(P<0.05)$. Arrows point to the values for HMOX1

of these genes (Fig. 3a), but 150 genes showed $>2$-fold expression differences (Table S2). Gene expression was very strongly correlated between the baseline and DAstimulated conditions $(R=0.993$; Fig. 3b). To better understand the biology of DA-responsive genes, we carried out gene ontology (GO) term enrichment analysis for 3756 genes with expression FCs $>1$ SD (1SD cut-off represents $\sim 20 \%$ expression change upon DA stimulation). About 1762 genes showed reduced expression and 1994 showed increased expression. GO-terms related to apoptosis and immune responses were enriched (Fig. 3c). 


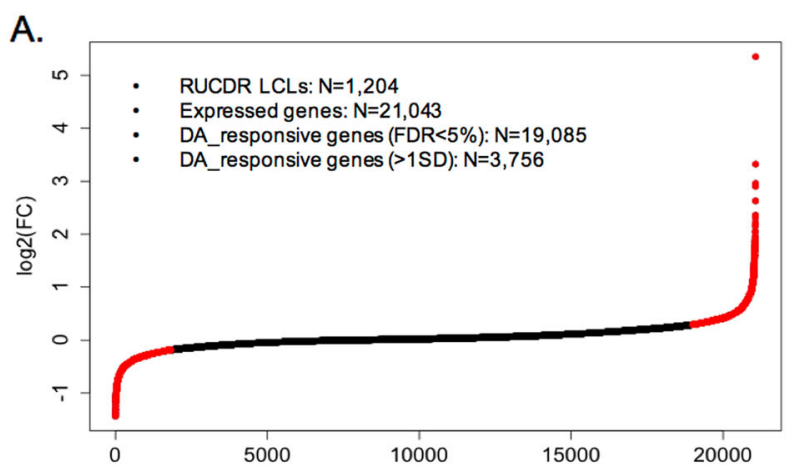

B.

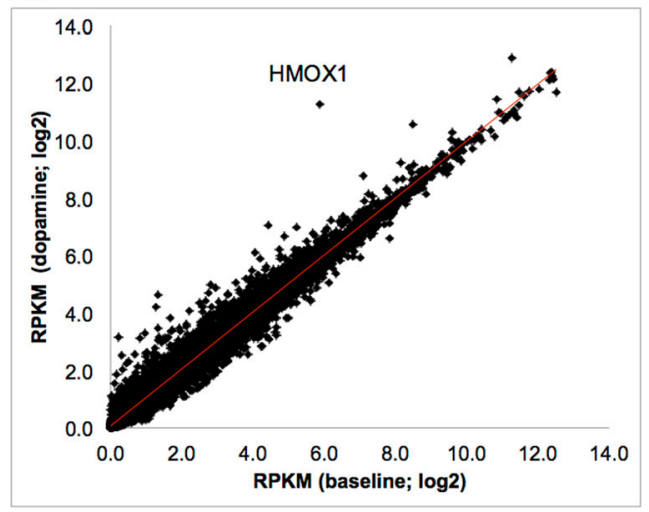

C.

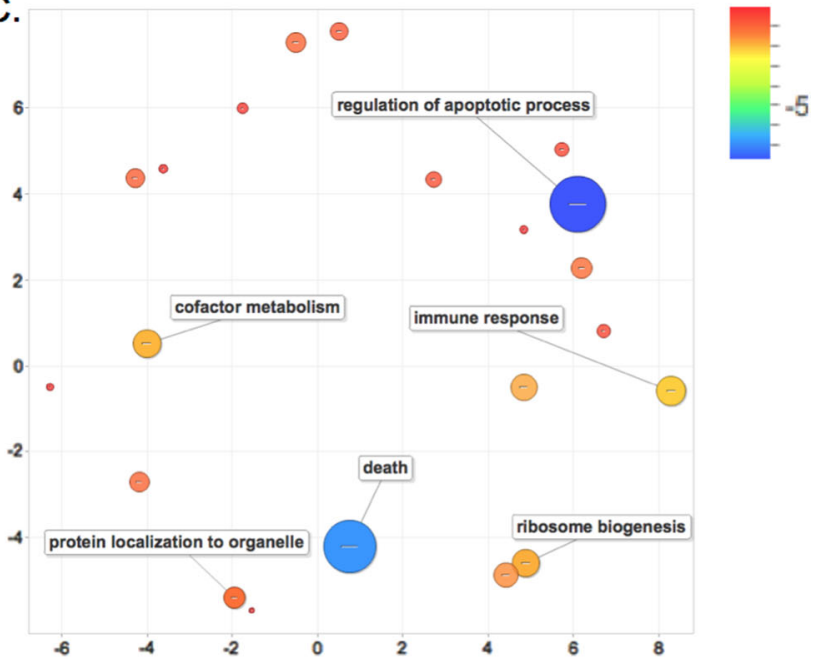

Fig. 3 DA-responsive genes in 1204 LCLs of MGS subjects. a Distribution of expression fold change (FC) for all genes expressed in LCLs upon DA $(100 \mu M)$ stimulation. An expressed gene was defined as having RPKM $>0$ in at least $50 \%$ of baseline or DA-stimulated samples. Two-side paired Student's t-test was used to test for DA response. The red dots show values for genes with log2 FC beyond the 1 SD cut off. b Gene expression before and after DA stimulation are highly correlated (Pearson $R=0.993$ ). c GO-term enrichment analysis by DAVID. The enriched GO-terms were clustered and visualized by REVIGO ${ }^{50}$. Scale bar $=\log (\mathrm{FDR})$, blue indicates the most significant enrichment

The most enriched GO-terms in the 1994 DAupregulated genes were related to apoptosis, while the most enriched GO-terms in the 1762 DA-downregulated genes were related to RNA processing and metabolism (Table S3). These results are in agreement with the hypothesis that DA stimulation had a widespread toxic effect leading to cell death and apoptosis, as previously described $^{59}$.

We examined whether genes showing relatively large FC were related to DA effects in the brain. Out of the five genes (HMOX1, GDF15, AMBP, SLC48A1, and NQO1) showing $>5$-fold expression changes (see Table S3), four were expressed at highly elevated levels (HMOX1, GDF15, $A M B P$, and NQO1) and have brain functions likely related to DA mechanisms. The most stimulated ( $>42$-fold) gene, HMOX1 (heme oxygenase 1), is hypothesized to induce pathological brain iron sequestration under oxidative stress $^{61}$ and has been reported to be upregulated by DA in cultured rat astrocytes, where astrocyte-specific proteins and pathways (e.g., impairment of glutamate transporters) play important roles in neurodegenerative diseases ${ }^{62}$. Furthermore, transgenic mice overexpressing $H M O X 1$ showed SZ-relevant features including increased tyrosine hydroxylase $(\mathrm{TH})$, augmented DA and serotonin levels in basal ganglia, and attenuated prepulse inhibition ${ }^{63}$. Interestingly, over-expression of HMOX1 in brain has been reported in Alzheimer's disease (AD), PD, multiple sclerosis (MS), and other degenerative and nondegenerative CNS diseases ${ }^{61,64,65}$. The second most stimulated (10-fold) gene, GDF15 (growth differentiation factor 15), encodes a trophic factor for midbrain DA neurons ${ }^{6,67}$. AMBP (alpha-1-microglobulin/bikunin precursor) has been proposed to be a urinary marker for major depression ${ }^{68}$, and NQO1 (NAD $(P) H$ dehydrogenase, quinone 1) encodes an enzyme which removes quinone, leading to protection of DA cells ${ }^{69-71}$. NQO1 has also been considered as a SZ candidate gene ${ }^{72}$, although unsupported by SZ-GWAS ${ }^{14}$. These results strongly 
suggest that the non-receptor mediated DA effects in LCLs may be relevant to brain disorders including SZ.

DA-mediated transcriptomic responses associated with SZ

We hypothesized that some DA-induced transcriptomic changes would differ in SZ cases and controls and used multiple linear regression analysis to test whether DAinduced expression FC was associated with disease status. Cell count and ATP level were correlated at both baseline and DA-stimulated conditions $(\mathrm{R}=0.58$ for both variables). We also examined analytical robustness by repeating the analysis omitting the cell counts and ATP levels obtained after DA stimulation as covariates, which yielded very similar results (Fig. S4). Overall, 1455 genes ( $\sim \%$ of all analyzed genes) showed SZ-associated differential DA response at FDR $<5 \%$ (Table S2). Among the top-ranking genes were interferon-induced protein with tetratricopeptide repeats 3 (IFIT3; rank \#2) and interferon receptor 1 (IFNAR1; rank \#15; Table S2), implying a possible pathogenic role of IFN signaling genes. Interestingly, IFIT3 and IFNAR1 have been recently implicated in blood transcriptome profiles of recurrent major depression $^{73}$. Also among the top-ranking genes showing SZ-associated differential DA response was tumor necrosis factor receptor superfamily, member $11 b$ (TNFRSF11b; rank \#1), which may be involved in TNF- $\alpha$-induced apoptosis $^{24}$. The most enriched GO-terms among genes showing SZ-associated differential expression response to DA stimulation were immune system process and response $\left(\mathrm{FDR}<1.1-1.3 \times 10^{-11}\right.$ ) as well as response to virus $\left(\mathrm{FDR}<1.99 \times 10^{-8}\right.$; Table S5). GO-terms related to regulation of apoptosis were also highly enriched (FDR < $3.41 \times 10^{-5}$; Table S5). Since it is possible that the chosen covariates did not sufficiently tag all existing confounders, we further carried out an exploratory analysis where we regressed out the effects of the top five expression PCs after accounting for the effects of the above-mentioned covariates. In spite of this analysis substantially reducing the total number of SZ-associated differential DAresponsive genes (from 1455 to 588; Table S2), the topranked genes and the most enriched GO-terms remained very similar (Table S6). Together with the observed inhibitory effect of DA on LCL growth, these results suggest that certain immune genes, including those related to apoptosis, may play a role in SZ pathogenesis and mediate the differential response to DA between cases and controls.

We then tested whether the set of genes showing differential DA response in SZ was enriched for genes that were: (1) brain expressed, (2) differentially expressed in schizophrenia postmortem brains, (3) loci showing GWS association to SZ, or (4) located in SZ-risk $\mathrm{CNVs}^{55}$. We found significant enrichment of brain-expressed genes (1.5-fold enrichment; $P=3.2 \times 10^{-96}$, two-sided Fisher's exact test). In the Stanley SZ postmortem brain collection (www.stanleygenomics.org) we observed enrichment of SZ-associated genes (1.8-fold enrichment; $P=3.3 \times 10^{-4}$, two-sided Fisher's exact test; Table 1). Furthermore, we found a non-significant enrichment of genes spanned by SZ-associated $\mathrm{CNVs}^{55}$ (1.4-fold enrichment; $P=0.25$, two-sided Fisher's Exact test; Table 1). We also observed a nominally significant enrichment of SZ-GWAS genes (1.8-fold enrichment, $P=0.03$, two-sided Fisher's Exact test; Table 1). Table S7 shows the 25 SZ-GWAS genes and $8 \mathrm{SZ}-\mathrm{CNV}$ genes that showed differential response to DA between SZ cases and controls. These genes include dihydropyrimidine dehydrogenase $(D P Y D)$ at $1 \mathrm{p} 21.3$, one of the loci showing the strongest association with SZ, although the strongest association signal there clusters around two microRNAs ${ }^{14}$, MIR137 and MIR2682, where we have found a rare functional enhancer SNP possibly associated with both $\mathrm{SZ}$ and bipolar disorder ${ }^{74}$. Four genes (MAPK3, SPN, TAOK2, and YPEL3) out of the 8 SZ-CNV genes that showed SZ-associated differential expression response to DA are in the 16p11.2 duplication $\mathrm{CNV}$, suggesting a possible role for DA dysfunction in the pathogenic mechanism underlying this CNV's association with SZ (Table S7).

\section{Comparison of case-control gene expression differences in baseline and DA-stimulated conditions}

We have recently reported genes that showed differential expression at the baseline (unstimulated) condition between SZ cases and controls in a meta-analysis of both RNAseq (complete overlap with this current study) and microarray data sets with similar sample size ${ }^{37}$. Here we examined whether genes showing SZ-associated differential DA response also showed case-control differential expression under baseline or DA-perturbed conditions. Using a similar linear regression analysis as described above, we tested the case-control gene expression differences at baseline and upon DA stimulation, respectively. When a pre-defined set of cell culture and demographic covariates was regressed out, we found that the SZassociated differentially expressed genes under both conditions showed a substantial overlap $(n=3655$; Fig. 4 a and Table S2), and the directions of case/control differential expression for the overlapping genes were completely concordant (except for one RNA gene ENSG00000278514.1) (Fig. S5). About 84\% of the genes showing SZ-associated differential DA response were also differentially expressed at baseline and/or DA-stimulation conditions (Fig. 4a). Genes that only showed SZassociated differential DA response (but did not differ at either baseline or after DA exposure) are most enriched for GO-terms related to apoptosis (Fig. 4b), while the rest are most enriched for GO-terms related to immune response (Fig. 4c). 

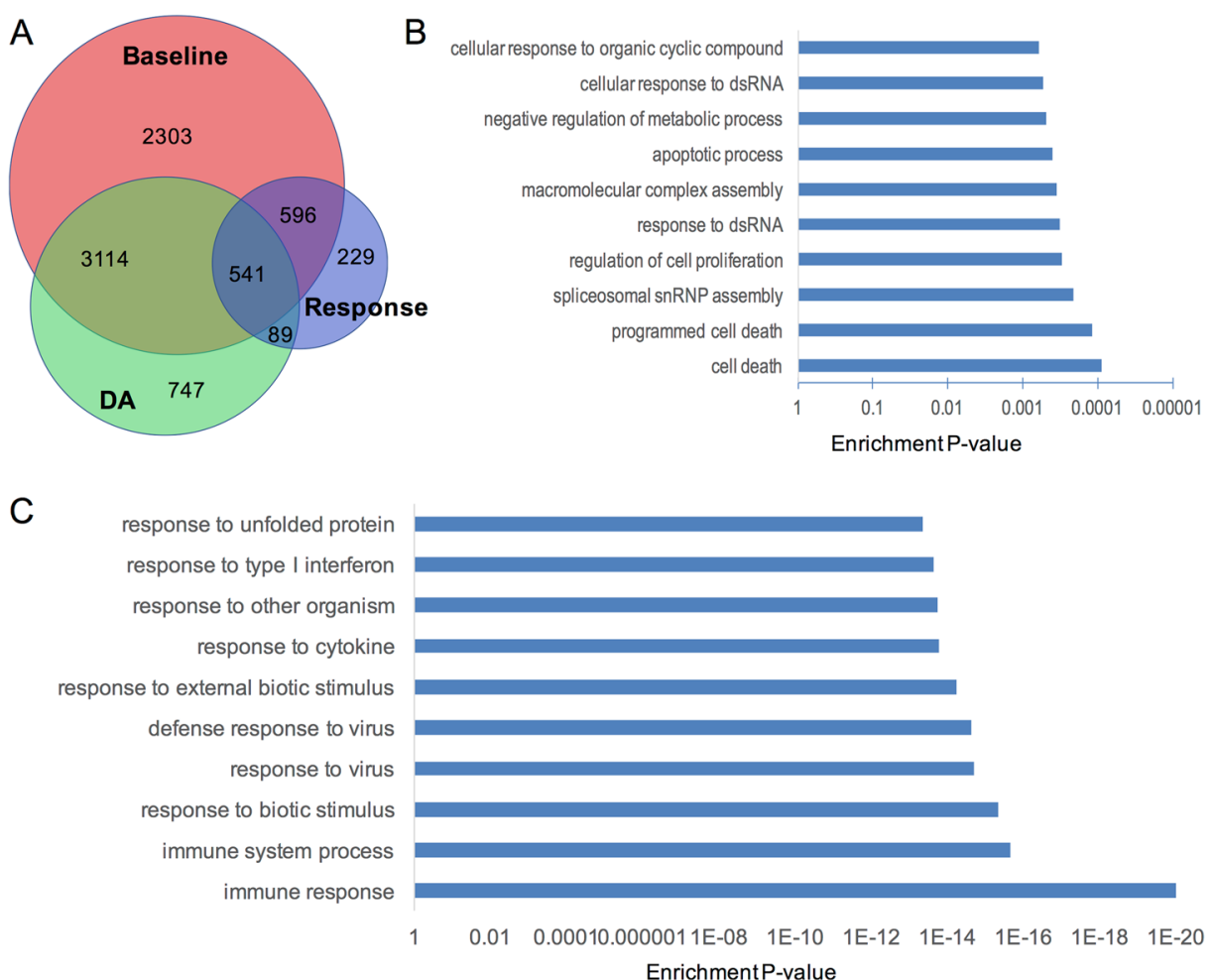

Fig. 4 Genes showing SZ-associated differential expression under different conditions and the enriched gene ontology terms. a Venn diagram that shows the overlap of genes showing SZ-associated differential DA response, differentially expressed genes in baseline and under DA stimulation. b 229 genes that only showed SZ-associated differential response to DA are highly enriched for GO-terms related to cell death. c 541 genes that showed SZ-associated differential expression in all three analyses are highly enriched for GO-terms related to immune response and response to virus

In our alternative model using both known covariates and the top five expression PCs, geared towards making sure that we adjust even for unknown potential confounders, we found a substantially smaller number of genes that showed SZ-associated differential expression under each condition (Table 1 and Fig. S6). Most (92\%) of the genes showing SZ-associated differential DA response under this analytical model were not differentially expressed at either baseline or DA-stimulation condition (Fig. S6). Similar to the genes showing SZ-associated differential DA response, genes that were differentially expressed in SZ cases under baseline or DA-perturbed conditions are also highly enriched for GO-terms involving immune response.

Enrichment analyses of baseline and DA-stimulated conditions yielded overlapping but different gene sets (Fig. 4). We found that differentially expressed genes in cases in the baseline condition (but not upon DA stimulation) showed a nominally significant enrichment for PGC2 SZ GWS genes $(P=0.04$, two-sided Fisher's Exact test). Furthermore, compared to genes differentially expressed in case/control in the baseline condition, genes showing SZ-associated differential DA response showed more fold enrichment of brain-expressed genes (1.5 vs. 1.3), SZCNV genes (1.4 vs. 0.8 ) and Stanley SZ-associated brainexpressed genes (1.8 vs. 1.4; Table 1). Interestingly, in an exploratory analysis where both known covariates and the top 5 expression PCs were regressed out, only genes showing SZ-associated differential DA response showed significant enrichment for brain-expressed genes and Stanley SZ-associated brain-expressed genes (Table 1). Finally, five SZ-CNV genes and four SZ-GWAS genes that showed SZ-associated differential DA response did not show SZ-associated differential expression under the baseline condition (Table S7). Our results suggest that DA perturbation was instrumental to detect relevant transcriptomic profiles missed by the analysis of baseline condition only.

\section{Discussion}

SZ-risk loci include both common and rare variants in many genes, each with small to modest effects on disease risk, as well as polygenic contributions of individually even smaller, and so far impossible to localize, effects ${ }^{75,76}$. We have studied the differential transcriptomic effects of DA perturbation of LCLs from SZ cases and controls, and 
found that a substantial number of genes responded to DA perturbation, that the DA response differed for many genes between SZ cases and controls, and that these differential response genes are enriched for immune and apoptosis-related genes, as well as those implicated by previous SZ-GWAS and CNV studies. Most genes showing SZ-associated differential expression under DA stimulation were also differentially expressed at baseline conditions (Figs. 3a \& 4). However, $16 \%$ of the genes showing differential response to DA by SZ case-control status did not show differential expression at the baseline condition. This suggests that relevant but cryptic biological mechanisms associated with schizophrenia become detectable in our model only by functional perturbation.

Although abnormal DA neurotransmission remains a major pathogenic hypothesis for SZ, transcriptional effects of DA perturbation and the possible differential DA response in a large sample of SZ cases and controls have not been previously investigated. The use of strong system perturbations to increase the size of functional effects of genetic variation (and therefore their detectability) is becoming more recognized ${ }^{77}$. In organisms or even in a simple cellular model, the inherent redundancy of regulatory molecular and cellular mechanisms, e.g., duplicated paralogs ${ }^{78}$, buffers biological systems for the effect of many genetic variations. Such systems' "robustness" thus may frequently mask the small effect sizes of common genetic variations even if they can cause disease under appropriate environmental stimuli. Our DA perturbation generates an artificial stress situation designed to mimic a relevant environmental stimulus as follows: an asymptomatic individual has a normal range of DA tone, and their DA-related networks only express variation which translates into normal physiological nonpathological states. However, as soon as this individual abuses amphetamine or cocaine (DA-releasing drugs associated with psychosis ${ }^{79}$ ) or upon exposure to other environmental SZ-risk factors, the activation of cryptic genetic variation may uncover an underlying genetic susceptibility to delusions and hallucinations, and the individual may then develop an episode of psychosis. By using LCLs derived from SZ cases and controls as a simplified albeit imperfect cellular model of SZ, our DA perturbation revealed transcriptomic changes that were invisible to the baseline transcriptomic study, adding additional information for understanding disease biology underlying the genetic contributions to SZ. Compared to genes showing SZ-associated differential expression in baseline or DA-stimulated conditions, genes showing differential DA responses between SZ cases and controls are more enriched for those implicated by SZ-GWAS, $\mathrm{CNV}$ studies, and postmortem brain transcriptomic studies (Table 1). Altogether, these results suggest that DA perturbation and testing case-control differential transcriptomic response to DA may add value to studying disease-relevant transcriptomic baseline profiles by enriching for disease-relevant genes.

Genes showing SZ- associated differential response to DA perturbation are highly enriched for those related to immune response (Fig. 3 and Table S4). In part, our results may be enabled by the type of tissue (i.e., immune) used for the experiments. However, we note that genes related to IFN and tumor necrosis factor (TNF) functions are among the top-ranking genes showing SZ-associated differential DA response (Table S3), many of which belong to interferon pathway genes (IFIT3, IFIT2, ISG15, $M X 2, O A S L$, and USP18) that have been previously implicated in in blood transcriptome profiles of recurrent major depression ${ }^{73}$. Our results overall are consistent with the immune hypothesis of $\mathrm{SZ}^{80,81}$. Maternal immune activation (e.g., via an infectious exposure such as influenza) has been reported to be associated with risk of SZ and autism, and blood levels of cytokines (e.g., IL1B, IL2RA, IL-6, and TNF) are elevated in SZ patients (see reviews ${ }^{82-84}$ ). Large population-based cohort research also suggests a shared etiology between SZ and several other autoimmune diseases, with increased risks of 1.1-1.6 for $\mathrm{SZ}^{80}$. In addition, epidemiological evidence shows a negative correlation between rheumatoid arthritis, an autoimmune disease, and $\mathrm{SZ}^{85}$. Most recent $\mathrm{SZ}$ GWAS also strongly suggest the involvement of immune mechanisms in SZ pathogenesis. The strongest and most replicable SZ GWAS finding is at the $\mathrm{xMHC}$ region ${ }^{14,17-}$ ${ }^{21}$. Furthermore, immune-related genes were enriched among the transcripts differentially expressed by SZ affection status in our previous baseline transcriptome profiling studies ${ }^{36,37}$. Cytokines play roles in cytotoxicity as well as influence DA and other neurotransmission systems that are implicated in the pathophysiology of $\mathrm{SZ}^{23}$. For instance, IL-6 modestly increases locomotion in rodents, behaviors modeling hyperdopaminergic-related psychotic symptoms in $\mathrm{SZ}^{86,87}$. Because of the possible role of cytokines and inflammatory factors in early-life infectious exposure of SZ patients, anti-inflammatory agents, such as celecoxib and aspirin, have been used as novel treatments in SZ patients to relieve some psychotic symptoms ${ }^{23}$. On the other hand, some antipsychotic drugs rebalance the immune response in SZ patients in microglial cells and astrocytes in the $\mathrm{CNS}^{25,26}$. Part of the mechanism is through modulating expression of cyclooxygenase-2 (COX-2), and growing evidence from clinical studies with COX-2 inhibitors points to favorable effects of anti-inflammatory therapy in $\mathrm{SZ}^{25,26}$.

DA stimulation had a widespread effect on gene expression in LCLs, leading to cell death likely through apoptosis as suggested from our gene set enrichment analysis. Consistently, we only observed the enrichment of apoptosis-related genes in the gene set showing increased 
expression upon DA stimulation. Apoptotic (and inflammatory) pathways are altered both in brain and the periphery during PD, a disease where neuronal loss is associated with chronic neuroinflammation characterized by microglial activation through the release of inflammatory mediators, as well as apoptosis triggered by the neuronal increase of calcium and $\mathrm{DA}^{59}$. Such neurodegenerative aspects (including, e.g., cellular apoptosis/ excitotoxicity) have been proposed to confer vulnerability to $\mathrm{SZ}^{25}$. Although we did not find robust expression of mRNAs of any DA receptors in LCLs (Table S1), a finding which was supported by the observed inability of DA antagonists to block DA effects on cell growth and gene expression changes (Fig. 2), our main observations appear to be consistent with reported DA effects in brains or in neuronal cell cultures. For instance, four (HMOX1, GDF15, $A M B P$, and NQO1) out of the five most highly DA-responsive genes are related to brain DA function. It has been controversial whether DA receptors are expressed in human peripheral blood lymphocytes ${ }^{88-92}$. Although we did not examine DA receptor expression at a protein level in our LCLs, the observed extremely low level of DA receptor mRNAs suggested our observed DA effects on cell growth and gene expression were likely mediated through non-receptor mediated mechanisms. A possible mechanism for DA function in LCLs may be through DA autoxidation, a process that contributes to DA neuron loss in PD or other neurodegenerative disorders involving DA neurotransmission ${ }^{15,16}$. Like in the human body, DA in cell culture may directly interact with oxygen, yielding quinones plus various free radicals as products $^{15,16}$. In support of this hypothesis, some of our observed highly DA-responsive and brain-functionrelevant genes are related to oxidation. For example, the most DA-responsive gene (HMOX1) contributes to iron sequestration under oxidative stress ${ }^{61}$, another highly DAresponsive gene (NQO1) catalyzes removal of the quinone ${ }^{69-71}$, and QPRT (quinolinate phosphoribosyltransferase) is one of the three genes within SZ-associated 16 p11.2 duplications that responded to DA perturbation. However, the roles of DA autoxidation in SZ pathophysiology remain to be further explored.

It is noteworthy that we have observed a widespread effect of DA on gene expression changes $(>90 \%$ of the genes) in LCLs and a very high percentage of genes that showed SZ-associated differential expression under baseline $(31 \%)$ or DA stimulation $(21 \%)$ conditions. We think that our observation is not a reflection of nonspecific effects of a seemingly high DA concentration or other technical artifacts, but rather a result of using a large number of well-controlled LCLs whose authentic biology is revealed by the experiment. First of all, the chosen DA concentration $100 \mu \mathrm{M}$ is within the range commonly used by the field ${ }^{56-58}$, which is even lower than the estimated DA concentration $(1.6 \mathrm{mM})$ in rat brain synaptic cleft ${ }^{60}$, and did not show much inhibitory effect on cell growth (Fig. 2a). Secondly, to minimize any possible effects of technical confounders, we have intercalated SZ cases and controls in each batch of cell culture, RNA preparation, and RNAseq. Moreover, our QC metrics did not show any systematic case/control bias or batch effects, and we have excluded three potential outlier samples identified by expression PCA and other QC procedures (Figs. S1-3). Instead, our large sample size may have contributed to the large number of differentially expressed genes by boosting the power to detect very small effects of DA on gene expression (only 150 genes showed $>2$-fold expression change upon DA stimulation). Biologically, DA affected cell growth, a central process that involves diverse signaling pathways, and it is thus unsurprising that we observed drastic transcriptomic changes upon DA stimulation. With regards to the large number of genes showing differential expression in SZ cases, our observation may be biologically explained by the association of $\mathrm{SZ}$ with immune-related genes including those in the $\mathrm{xMHC}$ region ${ }^{14,17-21}$. Indeed, there are 8 HLA genes (HLA-A, HLA-B, HLA-DMA, HLA-DOA, $H L A-D O B, H L A-D P B 1, H L A-D R A$, and $H L A-F)$ and 21 histone genes at the $\mathrm{xMHC}$ region that showed differential expression in SZ cases at baseline (Table S2).

Alternatively, detecting such large number of SZassociated differentially expressed genes in a wellpowered sample may reflect the "omnigenic" model, where most, if not all, genes outside core disease-related pathways are also involved in conferring disease liability by indirectly affecting the functions of core genes ${ }^{93}$. However, we have found that the number of genes showing SZ-associated differential DA response are much fewer than those differentially expressed at baseline or upon DA stimulation (Table 1). This might be explained by the fact that each LCL serves as an internal control when examining the differential DA response, where relevant (but potentially unknown) variables (including confounders) might play much less of a role than at baseline or DA-stimulated conditions.

The use of LCLs as a cellular model vs. brain (presumably the most relevant tissue for SZ) for DA perturbation in our study presents some clear limitations, because some gene expression changes in LCLs substantially differ from that in brain. However, LCLs are the most accessible tissue with a sizable sample, compared with other alternatives such as postmortem brain or neuronal cell lines, and also allow for experimental manipulations such as DA perturbation. Furthermore, a large proportion of gene expression signatures are shared between different tissues ${ }^{28-35}$, and we expect many LCLexpressed genes also show relevant transcription in the brain. Indeed, we have found a nominal enrichment of 
genes implicated by SZ-GWAS (Table 1), while a large SZ postmortem brain transcriptomic profiling study from the CommonMind Consortium (258 SZ cases and 279 controls) did not find enrichment of SZ-GWAS genes ${ }^{94}$. Another potential limitation of using LCLs as a model is that some functional effects may be an artifact of EBV transformation to produce the LCLs. This concern may be particular relevant for the observation that IFN and TNF pathway genes are among the most significantly SZassociated differential DA responses (Table S2). However, we have included EBV copy number as a covariate in the analyses (and also excluded monoclonal or pauciclonal LCLs); we thus expect that EBV copy number would pose minimal confounding effects on our observations. Therefore, regardless of LCLs not being brain-derived cells, given the strong support for an immune hypothesis of $\mathrm{SZ}^{95}$, our DA perturbation study on LCLs may provide some insights for immune aspects of SZ.

In summary, through DA perturbation that may be pathophysiologically relevant to SZ, we have demonstrated differential transcriptomic effects of DA in SZ cases and controls. Our results yield novel insight into SZ disease biology underlying SZ GWAS and CNV loci, and suggest a new approach to delineate the functional effect of genetic variants of small effect sizes by system perturbation.

\section{Acknowledgements}

We thank the study participants of MGS. MGS was mainly supported by R01MH059571, R01MH081800, and U01MH079469 (to P.V.G.); and other NIH grants for other MGS sites (R01MH067257 to N.G.B., R01MH059588 to B.J.M., R01MH059565 to R.F., R01MH059587 to F.A., R01MH060870 to W.F.B., R01MH059566 to D.W.B., R01MH059586 to J.M.S., R01MH061675 to D.F.L., R01MH060879 to C.R.C., U01MH046276 to C.R.C., and U01MH079470 to D.F.L). The transcriptomic work was primarily supported by RC2MH090030 (to A.R.S.), R01MH094116 (to H.H.H.G.), and R01MH094091 (to P.V.G.). Part of this work was conducted in facilities constructed under the support of $\mathrm{NIH}$ grant 1C06RR020547. This work was also supported by NorthShore University HealthSystem Research Career Development Award (to J.D.) and R01MH106575 (to J.D.).

\section{Author details}

${ }^{1}$ Center for Psychiatric Genetics, NorthShore University HealthSystem, Evanston, IL, USA. ${ }^{2}$ Department of Psychiatry and Behavioral Neuroscience, University of Chicago, Chicago, IL, USA. ${ }^{3}$ South Texas Diabetes and Obesity Institute, University of Texas Rio Grande Valley School of Medicine, San Antonio, TX, USA. ${ }^{4}$ Department of Genetics, Texas Biomedical Research Institute, San Antonio, TX, USA

\section{Conflict of interest}

The authors declare that they have no conflict of interest.

\section{Publisher's note}

Springer Nature remains neutral with regard to jurisdictional claims in published maps and institutional affiliations.

Supplementary Information accompanies this paper at (https://doi.org/ 10.1038/s41398-018-0216-5).
Published online: 16 August 2018

\section{References}

1. McGuffin, P., Farmer, A. E., Gottesman, I. I., Murray, R. M. \& Reveley, A. M. Twin concordance for operationally defined schizophrenia. Confirmation of familiality and heritability. Arch. Gen. Psychiatry 41, 541-545 (1984).

2. Carlsson, A. \& Lindqvist, M. Effect of chlorpromazine or haloperidol on formation of 3methoxytyramine and normetanephrine in mouse brain. Acta Pharmacol. Toxicol. 20, 140-144 (1963).

3. Creese, I., Burt, D. R. \& Snyder, S. H. Dopamine receptor binding predicts clinical and pharmacological potencies of antischizophrenic drugs. Science 192, 481-483 (1976).

4. Seeman, P. \& Lee, T. Antipsychotic drugs: direct correlation between clinical potency and presynaptic action on dopamine neurons. Science $\mathbf{1 8 8}$, 1217-1219 (1975).

5. Lieberman, J. A., Kane, J. M. \& Alvir, J. Provocative tests with psychostimulant drugs in schizophrenia. Psychopharmacol. (Berl.) 91, 415-433 (1987).

6. Snyder, S. H. The dopamine hypothesis of schizophrenia: focus on the dopamine receptor. Am. J. Psychiatry 133, 197-202 (1976).

7. Snyder, S. H. Proceedings: Drugs, neurotransmitters, and psychosis. Psychopharmacol. Bull. 10, 4-5 (1974).

8. Seeman, P. Dopamine receptors and the dopamine hypothesis of schizophrenia. Synapse 1, 133-152 (1987).

9. Howes, O. D. et al. The nature of dopamine dysfunction in schizophrenia and what this means for treatment. Arch. Gen. Psychiatry 69, 776-786 (2012).

10. Mita, T. et al. Decreased serotonin S2 and increased dopamine D2 receptors in chronic schizophrenics. Biol. Psychiatry 21, 1407-1414 (1986).

11. Abi-Dargham, A. et al. Increased striatal dopamine transmission in schizophrenia: confirmation in a second cohort. Am. J. Psychiatry 155, 761-767 (1998).

12. Laruelle, M. et al. Single photon emission computerized tomography imaging of amphetamine-induced dopamine release in drug-free schizophrenic subjects. Proc. Natl Acad. Sci. USA 93, 9235-9240 (1996).

13. Breier, A. et al. Schizophrenia is associated with elevated amphetamineinduced synaptic dopamine concentrations: evidence from a novel positron emission tomography method. Proc. Natl Acad. Sci. USA 94, 2569-2574 (1997).

14. PGC2. Biological insights from 108 schizophrenia-associated genetic loci. Nature 511, 421-427 (2014).

15. Segura-Aguilar, J. et al. Protective and toxic roles of dopamine in Parkinson's disease. J. Neurochem. 129, 898-915 (2014).

16. Meiser, J., Weindl, D. \& Hiller, K. Complexity of dopamine metabolism. Cell Commun. Signal. 11, 34 (2013).

17. Shi, J. et al. Common variants on chromosome 6p22.1 are associated with schizophrenia. Nature 460, 753-757 (2009).

18. International Schizophrenia C. et al. Common polygenic variation contributes to risk of schizophrenia and bipolar disorder. Nature 460, 748-752 (2009).

19. Stefansson, H. et al. Common variants conferring risk of schizophrenia. Nature 460, 744-747 (2009).

20. Schizophrenia Psychiatric Genome-Wide Association Study C. Genome-wide association study identifies five new schizophrenia loci. Nat. Genet. 43, 969-976 (2011).

21. Ripke, S. et al. Genome-wide association analysis identifies 13 new risk loci for schizophrenia. Nat. Genet. 45, 1150-1159 (2013).

22. Sekar, A. et al. Schizophrenia risk from complex variation of complement component 4. Nature 530, 177-183 (2016).

23. Girgis, R. R., Kumar, S. S. \& Brown, A. S. The cytokine model of schizophrenia: emerging therapeutic strategies. Biol. Psychiatry 75, 292-299 (2014).

24. Gierut, J. J. et al. Network-level effects of kinase inhibitors modulate TNF-alphainduced apoptosis in the intestinal epithelium. Sci. Signal. 8, ra129 (2015).

25. Muller, N., Myint, A. M., Krause, D., Weidinger, E. \& Schwarz, M. J. Antiinflammatory treatment in schizophrenia. Prog. Neuropsychopharmacol. Biol. Psychiatry 42, 146-153 (2013).

26. Muller, N., Myint, A. M. \& Schwarz, M. J. Immunological treatment options for schizophrenia. Curr. Pharm. Biotechnol. 13, 1606-1613 (2012).

27. Bialas, A. R. et al. Microglia-dependent synapse loss in type I interferonmediated lupus. Nature 546, 539-543 (2017).

28. Schadt, E. E. et al. Genetics of gene expression surveyed in maize, mouse and man. Nature 422, 297-302 (2003). 
29. Yan, H., Yuan, W., Velculescu, V. E., Vogelstein, B. \& Kinzler, K. W. Allelic variation in human gene expression. Science 297, 1143 (2002).

30. Cheung, V. G. et al. Natural variation in human gene expression assessed in lymphoblastoid cells. Nat. Genet. 33, 422-425 (2003).

31. Gretarsdottir, S. et al. The gene encoding phosphodiesterase 4D confers risk of ischemic stroke. Nat. Genet. 35, 131-138 (2003).

32. Dixon, A. L. et al. A genome-wide association study of global gene expression. Nat. Genet. 39, 1202-1207 (2007).

33. Emilsson, $\mathrm{V}$. et al. Genetics of gene expression and its effect on disease. Nature 452, 423-428 (2008).

34. Ioannidis, J. P. et al. Repeatability of published microarray gene expression analyses. Nat. Genet. 41, 149-155 (2009).

35. van Heerden, J. H. et al. Parallel changes in gene expression in peripheral blood mononuclear cells and the brain after maternal separation in the mouse. BMC Res. Notes 2, 195 (2009).

36. Sanders, A. R. et al. Transcriptome study of differential expression in schizophrenia. Hum. Mol. Genet. 22, 5001-5014 (2013).

37. Sanders, A. R. et al. Transcriptome sequencing study implicates immunerelated genes differentially expressed in schizophrenia: new data and a metaanalysis. Transl. Psychiatry 7, e1093 (2017).

38. Duan, J. et al. Transcriptome outlier analysis implicates schizophrenia susceptibility genes and enriches putatively functional rare genetic variants. Hum. Mol. Genet. 24, 4674-4685 (2015).

39. Levinson, D. F. et al. Copy number variants in schizophrenia: confirmation of five previous findings and new evidence for 3q29 microdeletions and VIPR2 duplications. Am. J. Psychiatry 168, 302-316 (2011).

40. Sanders, A. R. et al. The Internet-based MGS2 control sample: self report of mental illness. Am. J. Psychiatry 167, 854-865 (2010).

41. Choy, E. et al. Genetic analysis of human traits in vitro: drug response and gene expression in lymphoblastoid cell lines. PLoS Genet. 4, e1000287 (2008).

42. Bourne, J. A. SCH 23390: the first selective dopamine D1-like receptor antagonist. CNS Drug. Rev. 7, 399-414 (2001).

43. Sunahara, R. K. et al. Cloning of the gene for a human dopamine D5 receptor with higher affinity for dopamine than D1. Nature 350, 614-619 (1991).

44. Trapnell, C. et al. Differential gene and transcript expression analysis of RNAseq experiments with TopHat and Cufflinks. Nat. Protoc. 7, 562-578 (2012).

45. Anders, S. \& Huber, W. Differential expression analysis for sequence count data. Genome Biol. 11, R106 (2010).

46. Mortazavi, A., Williams, B. A., McCue, K., Schaeffer, L. \& Wold, B. Mapping and quantifying mammalian transcriptomes by RNA-Seq. Nat. Methods 5, 621-628 (2008).

47. $\mathrm{Li}, \mathrm{H}$. et al. The sequence alignment/map format and SAMtools. Bioinformatics 25, 2078-2079 (2009).

48. Huang da, W., Sherman, B. T. \& Lempicki, R. A. Systematic and integrative analysis of large gene lists using DAVID bioinformatics resources. Nat. Protoc. $\mathbf{4}$ 44-57 (2009)

49. Wang, J. \& Vasaikar, S. \& Shi, Z. \& Greer, M. \& Zhang, B. WebGestalt 2017: a more comprehensive, powerful, flexible and interactive gene set enrichment analysis toolkit. Nucleic Acids Res. 45, W130-W137 (2017).

50. Supek, F., Bosnjak, M., Skunca, N. \& Smuc, T. REVIGO summarizes and visualizes long lists of gene ontology terms. PLOS ONE 6, e21800 (2011).

51. Kang, H. J. et al. Spatio-temporal transcriptome of the human brain. Nature 478, 483-489 (2011)

52. Kirov, G. et al. Support for the involvement of large copy number variants in the pathogenesis of schizophrenia. Hum. Mol. Genet. 18, 1497-1503 (2009).

53. Stefansson, $\mathrm{H}$. et al. Large recurrent microdeletions associated with schizophrenia. Nature 455, 232-236 (2008).

54. International Schizophrenia C. Rare chromosomal deletions and duplications increase risk of schizophrenia. Nature 455, 237-241 (2008).

55. Marshall, C. R. et al. Contribution of copy number variants to schizophrenia from a genome-wide study of 41,321 subjects. Nat. Genet. 49, 27-35 (2017).

56. Dukes, A. A., Van Laar, V. S., Cascio, M. \& Hastings, T. G. Changes in endoplasmic reticulum stress proteins and aldolase $A$ in cells exposed to dopamine. J. Neurochem. 106, 333-346 (2008).

57. Duan, J. et al. Synonymous mutations in the human dopamine receptor D2 (DRD2) affect mRNA stability and synthesis of the receptor. Hum. Mol. Genet. 12, 205-216 (2003).

58. Rohr, O., Sawaya, B. E., Lecestre, D., Aunis, D. \& Schaeffer, E. Dopamine stimulates expression of the human immunodeficiency virus type 1 via NFkappaB in cells of the immune system. Nucleic Acids Res. 27, 3291-3299 (1999).
59. Macchi, B. et al. Inflammatory and cell death pathways in brain and peripheral blood in Parkinson's disease. CNS Neurol. Disord. Drug. Targets 14, 313-324 (2015).

60. Garris, P. A., Ciolkowski, E. L., Pastore, P. \& Wightman, R. M. Efflux of dopamine from the synaptic cleft in the nucleus accumbens of the rat brain. J. Neurosci. 14, 6084-6093 (1994).

61. Schipper, H. M. Heme oxygenase-1: transducer of pathological brain iron sequestration under oxidative stress. Ann. N Y Acad. Sci. 1012, 84-93 (2004).

62. Maragakis, N. J. \& Rothstein, J. D. Mechanisms of disease: astrocytes in neurodegenerative disease. Nat. Clin. Pract. Neurol. 2, 679-689 (2006).

63. Song, W. et al. Schizophrenia-like features in transgenic mice overexpressing human HO-1 in the astrocytic compartment. J. Neurosci. 32, 10841-10853 (2012).

64. Schipper, H. M. Heme oxygenase-1: role in brain aging and neurodegeneration. Exp. Gerontol. 35, 821-830 (2000).

65. Schipper, H. M. Heme oxygenase expression in human central nervous system disorders. Free Radic. Biol. Med. 37, 1995-2011 (2004).

66. Strelau, J. et al. Growth/differentiation factor-15/macrophage inhibitory cytokine-1 is a novel trophic factor for midbrain dopaminergic neurons in vivo. J. Neurosci. 20, 8597-8603 (2000).

67. Strelau, J., Schober, A., Sullivan, A., Schilling, L. \& Unsicker, K. Growth/differentiation factor-15 (GDF-15), a novel member of the TGF-beta superfamily, promotes survival of lesioned mesencephalic dopaminergic neurons in vitro and in vivo and is induced in neurons following cortical lesioning. J. Neural Transm. Suppl. 65, 197-203 (2003).

68. Wang, Y. et al. Urinary peptidomics identifies potential biomarkers for major depressive disorder. Psychiatry Res. 217, 25-33 (2014).

69. Choi, H. J., Kim, S. W., Lee, S. Y. \& Hwang, O. Dopamine-dependent cytotoxicity of tetrahydrobiopterin: a possible mechanism for selective neurodegeneration in Parkinson's disease. J. Neurochem. 86, 143-152 (2003).

70. Han, J. M. et al. Protective effect of sulforaphane against dopaminergic cell death. J. Pharmacol. Exp. Ther. 321, 249-256 (2007).

71. Son, H. J. et al. Induction of NQO1 and neuroprotection by a novel compound KMS04014 in Parkinson's disease models. J. Mol. Neurosci. 56, 263-272 (2015).

72. Hori, H., Ohmori, O., Matsumoto, C., Shinkai, T. \& Nakamura, J. NAD(P)H: quinone oxidoreductase (NQO1) gene polymorphism and schizophrenia. Psychiatry Res. 118, 235-239 (2003).

73. Mostafavi, S. et al. Type I interferon signaling genes in recurrent major depression: increased expression detected by whole-blood RNA sequencing. Mol. Psychiatry 19, 1267-1274 (2014).

74. Duan, J. et al. A rare functional noncoding variant at the GWAS-implicated MIR137/MIR2682 locus might confer risk to schizophrenia and bipolar disorder. Am. J. Hum. Genet. 95, 744-753 (2014).

75. Gottesman, I. I. \& Shields, J. A polygenic theory of schizophrenia. Proc. Natl Acad. Sci. USA 58, 199-205 (1967).

76. Lee, S. H. et al. Estimating the proportion of variation in susceptibility to schizophrenia captured by common SNPs. Nat. Genet. 44, 247-250 (2012).

77. Hermisson, J. \& Wagner, G. P. The population genetic theory of hidden variation and genetic robustness. Genetics 168, 2271-2284 (2004).

78. Dean, E. J., Davis, J. C., Davis, R. W. \& Petrov, D. A. Pervasive and persistent redundancy among duplicated genes in yeast. PLoS Genet. 4, e1000113 (2008).

79. Nissbrandt, H., Engberg, G., Wikstrom, H., Magnusson, T. \& Carlsson, A. NSD 1034: an amino acid decarboxylase inhibitor with a stimulatory action on dopamine synthesis not mediated by classical dopamine receptors. NaunynSchmiedeberg's Arch. Pharmacol. 338, 148-161 (1988).

80. Eaton, W. W., Pedersen, M. G., Nielsen, P. R. \& Mortensen, P. B. Autoimmune diseases, bipolar disorder, and non-affective psychosis. Bipolar Disord. 12 638-646 (2010).

81. Khandaker, G. M. \& Dantzer, R. Is there a role for immune-to-brain communication in schizophrenia? Psychopharmacol. (Berl.) 233, 1559-1573 (2016).

82. DeLisi, L. E. \& Crow, T. J. Is schizophrenia a viral or immunologic disorder? Psychiatr. Clin. North Am. 9, 115-132 (1986).

83. Knuesel, I. et al. Maternal immune activation and abnormal brain development across CNS disorders. Nat. Rev. Neurol. 10, 643-660 (2014).

84. Brown, A. S. \& Derkits, E. J. Prenatal infection and schizophrenia: a review of epidemiologic and translational studies. Am. J. Psychiatry 167, 261-280 (2010).

85. Lee, S. H. et al. New data and an old puzzle: the negative association between schizophrenia and rheumatoid arthritis. Int. J. Epidemiol. 44, 1706-1721 (2015).

86. Zalcman, S., Murray, L., Dyck, D. G., Greenberg, A. H. \& Nance, D. M. Interleukin2 and -6 induce behavioral-activating effects in mice. Brain Res. 811, 111-121 (1998). 
87. Zalcman, S., Savina, I. \& Wise, R. A. Interleukin-6 increases sensitivity to the locomotor-stimulating effects of amphetamine in rats. Brain Res. 847, 276-283 (1999).

88. Ricci, A. et al. Dopamine D1-like receptor subtypes in human peripheral blood lymphocytes. J. Neuroimmunol. 96, 234-240 (1999).

89. Takahashi, N., Nagai, Y., Ueno, S., Saeki, Y. \& Yanagihara, T. Human peripheral blood lymphocytes express D5 dopamine receptor gene and transcribe the two pseudogenes. FEBS Lett. 314, 23-25 (1992).

90. Vile, J. M. \& Strange, P. G. D2-like dopamine receptors are not detectable on human peripheral blood lymphocytes. Biol. Psychiatry 40, 881-885 (1996).

91. Vile, J. M. \& Strange, P. G. High-affinity binding sites for neuroleptic drugs in human peripheral blood lymphocytes and their relation to dopamine receptors. A long-standing controversy. Biochem. Pharmacol. 49, 747-753 (1995).

92. Bondy, B., de Jonge, S., Pander, S., Primbs, J. \& Ackenheil, M. Identification of dopamine D4 receptor mRNA in circulating human lymphocytes using nested polymerase chain reaction. J. Neuroimmunol. 71, 139-144 (1996).

93. Boyle, E. A., Li, Y. I. \& Pritchard, J. K. An expanded view of complex traits: from polygenic to omnigenic. Cell 169, 1177-1186 (2017).

94. Fromer, M. et al. Gene expression elucidates functional impact of polygenic risk for schizophrenia. Nat. Neurosci. 19, 1442-1453 (2016).

95. Consortium NaPASoPG. Psychiatric genome-wide association study analyses implicate neuronal, immune and histone pathways. Nat. Neurosci. 18, 199-209 (2015).

\section{MGS}

P. V. Gejman', A. R. Sanders ${ }^{5}$, J. Duann ${ }^{5}$, D. F. Levinson ${ }^{6}$, J. Shi ${ }^{7}$, N. G. Buccola ${ }^{8}$, B. J. Mowry ${ }^{9}$, R. Freedman ${ }^{10}$, A. Olincy ${ }^{10}$, F. Amin ${ }^{11}$, D. W. Black ${ }^{12}$, J. M. Silverman ${ }^{13}$, W. F. Byerley ${ }^{14}$, C. R. Cloninger ${ }^{15}$ \& D. M. Svrakic ${ }^{15}$

${ }^{5}$ NorthShore University HealthSystem and University of Chicago, Chicago, IL, USA. ${ }^{6}$ Stanford University, Stanford, CA, USA. ${ }^{7}$ National Cancer Institute, Rockville, MD, USA. ${ }^{8}$ Louisiana State University Health Sciences Center, New Orleans, LA, USA. ${ }^{9}$ Queensland Centre for Mental Health Research, Brisbane and Queensland Brain Institute, The University of Queensland, Brisbane, Australia. ${ }^{10}$ University of Colorado, Denver, CO, USA. ${ }^{11}$ Atlanta Veterans Affairs Medical Center and Emory University, Atlanta, GA, USA. ${ }^{12}$ University of lowa Carver College of Medicine, lowa, IA, USA. ${ }^{13}$ Mount Sinai School of Medicine, New York, NY, USA. ${ }^{14}$ University of California, San Francisco, CA, USA. ${ }^{15}$ Washington University, St. Louis, MO, USA 